\section{Placement control by transabdominal ultrasound of duodenal feeding tubes: a feasible alternative for trials in a healthy volunteer study population}

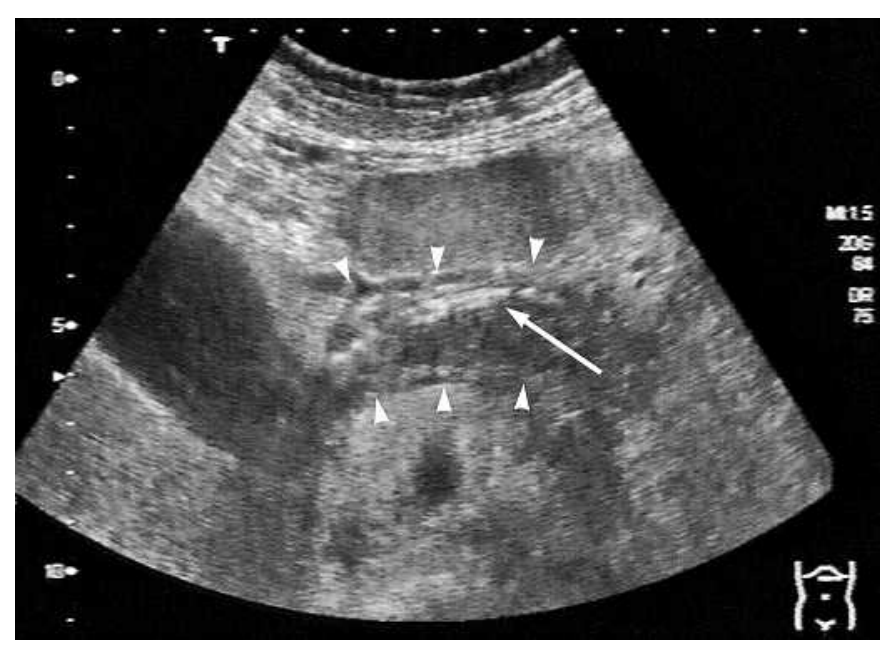

Figure 1 Pars III duodeni marked by arrowheads in the middle below the left liver lobe. Arrow indicates the feeding tube.

radiological methods for controlling tube position and for insertion is widely accepted for both patients and healthy volunteers. However, radiological methods remain controversial [1]. They carry radiation burden, which depends on the experience of the investigator and on anatomic conditions. Their use in healthy volunteers should therefore follow careful ethical consideration, and alternative methods should be sought if necessary. In critically ill patients, sonographically guided enteric feeding tube position placement at the bedside has already been successfully employed [2]. Hence, in this prospective study we investigated the use of ultrasonography to confirm correct intraduodenal tube placement on three occasions in 18 healthy volunteers $(\mathrm{m} / \mathrm{f} 0.8$; age $($ mean $\pm \mathrm{SD}) 27.8 \pm 5.4$ years; BMI $22.27 \pm 1.88 \mathrm{~kg} / \mathrm{m}^{2}$ ), leading to a total of 54 tube insertions. After an overnight fast and ingestion of $180 \mathrm{~mL}$ water, a polyurethane enteric feeding tube (diameter $2.6 \mathrm{~mm}$, length $120 \mathrm{~cm}$ ) was blindly inserted in right decubitus position. The manoeuvre was performed under continuous aspiration of gastrointestinal juice. The required location of the tube was verified by bedside measurement of aspirated fluid $\mathrm{pH}$ ( $\mathrm{pH} 6.9 \pm 0.9$ ). Transabdominal ultrasound (3.75-MHz Probe) visualized the enteral tube from the pylorus to the second part of the duodenum (mediolateral of the gallbladder and upper transverse section in front of the inferior vena cava), both in upright and supine positions (Figure 1). Visual detection was enhanced by injection of $10 \mathrm{~mL}$ air followed by $10 \mathrm{~mL}$ saline (Figure 2 ). In $98 \%$ of all insertions the tube was localized in the second part of the duodenum by ultrasonography. We believe ultrasonography is a feasible alternative to radiological methods for monitoring postpyloric tube placement, and is preferable for studies in populations of healthy volunteers.

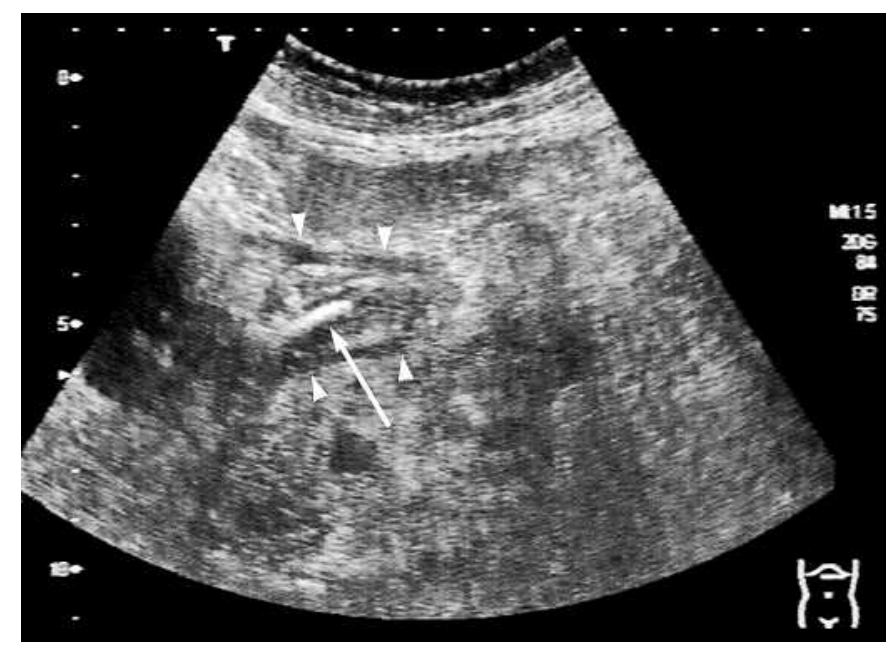

Figure 2 Injected hand-shaken air bubbles generate stronger scattering signals within the feeding tube (arrow).
Endoscopy_UCTN_Code_TTT_1AO_2AK

\section{O. Goetze, M. Fried, C. Gubler}

Division of Gastroenterology and Hepatology, Department of Internal medicine, University Hospital of Zurich, Switzerland.

\section{References}

${ }^{1}$ Hallstrom CK, Jacobs BR. Optimal method of postpyloric tube placement still unclear. Crit Care Med 2003; 31: 315-316

2 Hernandez-Socorro CR, Marin J, Ruiz-Santana $S$ et al. Bedside sonographic-guided versus blind nasoenteric feeding tube placement in critically ill patients. Crit Care Med 1996; 24: 1690-1694

\section{Corresponding author}

\section{Gubler, MD}

Division of Gastroenterology and Hepatology

Department of Internal Medicine University Hospital Zürich

Rämistrasse 100

8091 Zürich

Switzerland

Fax: +41-44-255-4503

Email: Christoph.Gubler@spitaluster.ch 